Claremont Colleges

Scholarship@ Claremont

All HMC Faculty Publications and Research

HMC Faculty Scholarship

8-1-1998

\title{
Ultrafast Phenomena: A Laboratory Experiment for Undergraduates
}

Thomas D. Donnelly

Harvey Mudd College

Carl Grossman

Swarthmore College

\section{Recommended Citation}

T.D. Donnelly and C. Grossman, "Ultrafast Phenomena: A Laboratory Experiment for Undergraduates," Am. J. Phys. 66, 677 (1998).

This Article is brought to you for free and open access by the HMC Faculty Scholarship at Scholarship @ Claremont. It has been accepted for inclusion in All HMC Faculty Publications and Research by an authorized administrator of Scholarship @ Claremont. For more information, please contact scholarship@cuc.claremont.edu. 


\section{AAPT AMERICAN \\ JOURNAL}

\section{Ultrafast phenomena: A laboratory experiment for undergraduates}

T. D. Donnelly and Carl Grossman

Citation: Am. J. Phys. 66, 677 (1998); doi: 10.1119/1.18932

View online: http://dx.doi.org/10.1119/1.18932

View Table of Contents: http://ajp.aapt.org/resource/1/AJPIAS/v66/i8

Published by the American Association of Physics Teachers

\section{Related Articles}

Improving the quantification of Brownian motion

Am. J. Phys. 81, 485 (2013)

Photon charge experiment

Am. J. Phys. 81, 436 (2013)

Collimated blue light generation in rubidium vapor

Am. J. Phys. 81, 442 (2013)

The Wiimote on the Playground

Phys. Teach. 51, 272 (2013)

Helicopter Toy and Lift Estimation

Phys. Teach. 51, 310 (2013)

\section{Additional information on Am. J. Phys.}

Journal Homepage: http://ajp.aapt.org/

Journal Information: http://ajp.aapt.org/about/about_the_journal

Top downloads: http://ajp.aapt.org/most_downloaded

Information for Authors: http://ajp.dickinson.edu/Contributors/contGenlnfo.html

\section{ADVERTISEMENT}

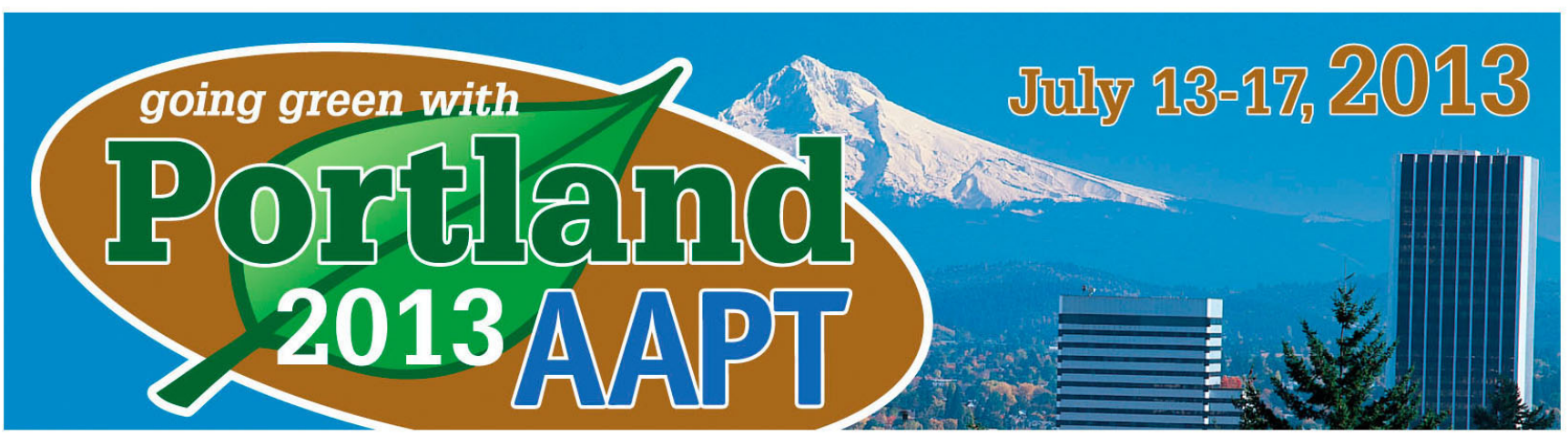




\section{Ultrafast phenomena: A laboratory experiment for undergraduates}

T. D. Donnelly a) and Carl Grossman

Department of Physics, Swarthmore College, Swarthmore, Pennsylvania 19081

(Received 12 June 1997; accepted 26 January 1998)

We present a set of experiments that introduce students to ultrafast science. We discuss the relationship between the description of an ultrashort laser pulse in the frequency domain and the time domain. Using experimental results we demonstrate that this relationship is constrained by the lower limit of the Heisenberg uncertainty principle. Students carrying out the experiments will become familiar with ultrafast techniques, such as autocorrelation and laser cavity design, as well as various other concepts such as dispersion, Fourier transformation, interference, and nonlinear optics.

C) 1998 American Association of Physics Teachers.

\section{INTRODUCTION}

"Ultrafast" is a term typically used to describe processes which occur on a picosecond $\left(10^{-12} \mathrm{~s}\right)$ or faster time scale. Up to about 1965 the shortest time interval that could be resolved was $1 \mathrm{~ns}\left(10^{-9} \mathrm{~s}\right)$, a limit set by the capabilities of high frequency electronic circuitry. A century earlier, time resolution of only milliseconds was available from streakrecording methods. ${ }^{1}$ The time resolution limit dropped precipitously after 1965 due to the invention of the laser and now is on the order of $10 \mathrm{fs}\left(10^{-14} \mathrm{~s}\right) .^{2,3}$

The techniques and technology of ultrafast science provide a unique method for investigating physical phenomena throughout the physical and biological sciences. ${ }^{4}$ For ex- ample, in 1991 Schoenlein et al. ${ }^{5}$ measured a 200 -fs time constant for the 11-cis to 11-trans torsional isomerization of the rhodopsin chromophore, the first step in the vision process. Investigations of photosynthesis ${ }^{6}$ showed the lifetime of the excited chlorophyll $\mathrm{b}$ molecule to be $15 \mathrm{ps}$ at concentrations near those of living cells $(0.1 \mathrm{M})$; these measurements can be used to infer the structure of the chlorophyll $b$ molecule. ${ }^{7}$ A number of DNA studies have also been carried out, some, for instance, studying the transfer of energetic excitations along the DNA molecule. ${ }^{8}$ Recently, the first study of protein dynamics using picosecond infrared vibrational echo experiments was carried out by Rella et al. ${ }^{9}$ They studied myoglobin-CO (used in storage and transport of $\mathrm{O}_{2}$ in muscle tissue) and showed the existence of many confor- 
mational states at physiologically relevant temperatures, thus providing insights into how protein dynamics are transmitted to the active site of myoglobin.

Ultrafast phenomena are also widely studied in physics and chemistry. Recently, high order harmonic generation has been studied in great detail. ${ }^{10}$ In this process a high intensity laser pulse $\left(10^{13}-10^{15} \mathrm{~W} / \mathrm{cm}^{2}\right)$ interacts with an atom to produce radiation at up to the 109th harmonic of the fundamental, optical-frequency pulse. ${ }^{11}$ As another example, the generation of picosecond $\mathrm{X}$-ray pulses has also been investigated. ${ }^{12}$ Here, a high intensity light pulse $\left(10^{16} \mathrm{~W} / \mathrm{cm}^{2}\right)$ irradiates a metal, thus creating a hot, dense plasma. The plasma is hot enough to produce $\mathrm{x}$ rays, yet cools so fast that the x-ray emission lasts for only 1 ps. Putterman and co-workers have used 200-fs laser pulses to temporally resolve the supersonic collapse of sonoluminescing bubbles. ${ }^{13}$ They have shown that the enigmatic light emission of an acoustically driven bubble occurs within 500 ps of the minimum bubble radius, that the bubble collapses with an acceleration greater than $10^{11} \mathrm{~g}$, and that the speed of the collapse is greater than Mach 4. Femtosecond lasers have also been used in various pump-probe configurations to study the carrier dynamics of semiconductors, ${ }^{14}$ molecular dynamics in liquids, ${ }^{15}$ and excitation dynamics in polymers. ${ }^{16}$ Ultrafast phenomena are also widely investigated in electrical engineering laboratories and industrial research centers. This is largely because ultrafast technology makes possible high data transfer rates in communication systems. $^{17}$

To date, there has been little development of ultrafast laboratories aimed at an undergraduate audience, and we attribute this to the relative expense of purchasing an ultrafast laser system. Recently, however, prices for femtosecond laser systems have dropped to the level where an institution, already in possession of an argon ion laser, can initiate a program for as little as $\$ 20,000$ (references to manufacturers and current prices are made in the experimental section). Using one such system, we have developed experiments with three goals in mind: the first is to teach students about ultrafast techniques and technology; the second is to create straightforward studies which can be linked to the fundamentals of quantum mechanics; the third is to develop experiments which can be carried out by undergraduates and are cost effective enough to be supported by an undergraduate institution. In carrying out the experiments students gain research experience which is relevant to both the academic and business communities.

\section{THEORY}

The Heisenberg uncertainty principle (HUP) states that there is a limit to the information we can acquire about conjugate variables of a system. For example, our simultaneous knowledge of both the position, $x$, and momentum, $p$, of a system is limited:

$$
\Delta x \Delta p \geqslant \hbar / 2 \text {. }
$$

Here, " $\Delta$ ', indicates the "uncertainty" in the value of the quantity being measured. Mathematically,

$$
\Delta x=\sqrt{\left\langle x^{2}\right\rangle-\langle x\rangle^{2}},
$$

where the brackets define an expectation value which is given by

$$
\langle x\rangle=\frac{\langle\Psi|x| \Psi\rangle}{\langle\Psi \mid \Psi\rangle}=\frac{\int_{-\infty}^{\infty} \Psi^{*} x \Psi d x}{\int_{-\infty}^{\infty} \Psi^{*} \Psi d x}
$$

A similar relation holds for the energy, $E$, and time, $t$, variables of a system:

$$
\Delta E \Delta t \geqslant \hbar / 2 \text {. }
$$

For photons $\Delta E=\hbar \Delta \omega$, so Eq. (4) can be rewritten as

$$
\Delta \omega \Delta t \geqslant 1 / 2 \text {. }
$$

In the context of a laser pulse, $\Delta \omega$ refers to the spectral bandwidth of the pulse and $\Delta t$ describes the pulse's temporal duration. It should be recalled that implicit in the word "pulse" is the idea of localization. For a pulse (i.e., a statistically large group of photons) to be localized in time it must contain many frequency components, as is known from Fourier decomposition, or, more qualitatively, from Eq. (5). In fact, the localization of a group of photons in an interval of time necessarily sets a limit to the minimum probable range of photon energies. The theoretical basis for this relation derives from the most general form of the Heisenberg uncertainty principle ${ }^{18}$

$$
\Delta A^{2} \Delta B^{2} \geqslant\left|\frac{1}{2}\langle[\hat{A}, \hat{B}]\rangle\right|^{2} \text {. }
$$

The time evolution of an expectation value is given by ${ }^{19}$

$$
\frac{d}{d t}\langle A\rangle=\frac{i}{\hbar}\langle[\hat{H}, \hat{A}]\rangle+\left\langle\frac{\partial}{\partial t} \hat{A}\right\rangle .
$$

The position-momentum uncertainty relation follows directly from Eq. (6). However, deriving the energy-time uncertainty relation is not as straightforward since $\hat{t}$ is not a valid operator. Even so, an expression analogous to $\Delta x \Delta p$ $\geqslant \hbar / 2$ can be developed for energy and time as follows. For an operator that does not depend explicitly on time $[(\partial / \partial t) \hat{A}=0]$ we can rewrite the general form of the uncertainty relation. Taking $\hat{B}=\hat{H}$, and using Eq. (7), we can rewrite Eq. (6) as

$$
\Delta E\left(\frac{\Delta A}{\left|\frac{d}{d t}\langle A\rangle\right|}\right) \geqslant \frac{\hbar}{2} .
$$

The term in the brackets can be identified as time uncertainty, $\Delta t$, if we interpret $\Delta t$ as a measure of the time it takes for the expectation value of observable $A$ to change significantly. ${ }^{20}$ For example, $\Delta t$ for a laser pulse with a Gaussian spatial profile may be considered as the amount of time it takes for the position of the pulse to change by the spatial width of the pulse, $\sigma_{x}$. Thus we arrive at the energytime uncertainly relation in Eq. (4) taking

$$
\left(\frac{\Delta A}{\left|\frac{d}{d t}\langle A\rangle\right|}\right) \approx \Delta t .
$$

Looking at Eq. (5) it is clear that a laser pulse of shorter temporal duration can be created if more spectral bandwidth is added to the pulse. To generate short pulses, therefore, we need a lasing medium which can supply a large gain bandwidth and a laser cavity which can support that bandwidth. The most widely used medium for supplying the bandwidth is titanium-doped sapphire $\left(\mathrm{Ti}: \mathrm{Al}_{2} \mathrm{O}_{3}\right)$ which lases over the range of approximately $700-1000 \mathrm{~nm} .^{21}$ This material can 


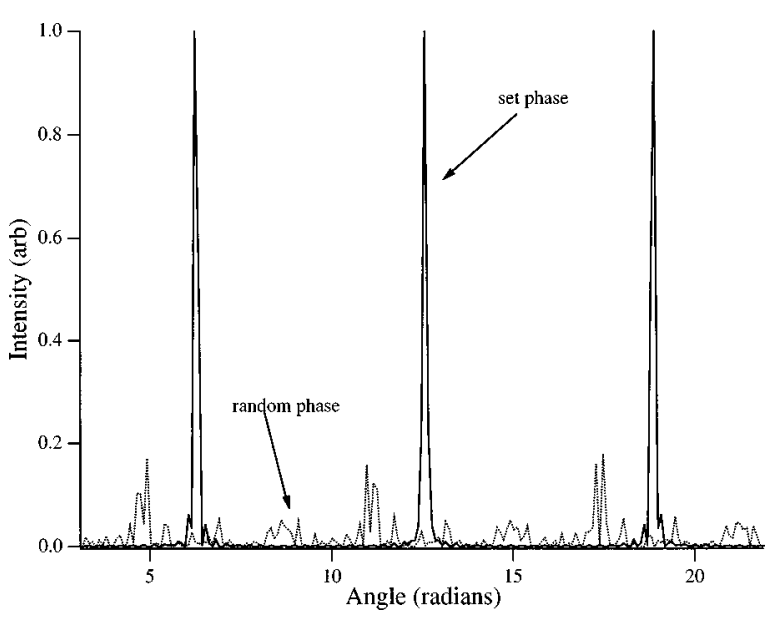

Fig. 1. The result of 20 cavity modes added together either with a fixed phase for each mode, or a random phase relationship. Note that a pulse is generated when the modes are "locked."

provide gain to over $10^{6}$ longitudinal cavity modes for a typical laser cavity. (The $n$th longitudinal cavity mode has wavelength $2 L / n$, where $L$ is the length of the laser cavity and $n$ is an integer. The $i$ th mode can be described mathematically as $A \sin \left(n_{i} \pi z / L+\phi_{i}\right)$, where $A_{i}$ is the amplitude and $\phi_{i}$ is the phase at $\left.z=0\right)$. This can be compared to a helium-neon laser which has a gain bandwidth on the order of $10^{-3} \mathrm{~nm}$ and therefore lases in only a few longitudinal cavity modes.

A large spectral bandwidth, however, is not a sufficient condition for ultrashort pulse generation. Each spectral component (longitudinal mode) must have a specific phase relation to the other components, i.e., the pulse must be "mode locked." This is illustrated in Fig. 1, a simulation of the pulse intensity resulting from the addition of 20 cavity modes. In one case, a random phase is assigned to each mode before the modes are added together and the result is a relatively flat intensity versus time. In fact, the intensity versus time tends toward a constant value as the number of randomphase cavity modes is increased. Alternatively, when a particular phase relation is set among the modes (e.g., the phase is zero for each wave in this case, although any constant phase would suffice) the sum results in very pronounced intensity spikes-we have created short pulses. The pulse is said to be "mode locked," as each of the modes has a specific phase relationship to one another.

In practice, mode locking the $\mathrm{Ti}: \mathrm{Al}_{2} \mathrm{O}_{3}$ pulse is achieved through Kerr-lens mode locking, a process which exploits the optical Kerr effect. ${ }^{22}$ Kerr-lens mode locking is utilized in a laser cavity designed to have higher gain for a pulse that has a short duration versus one of longer duration. This is possible because a short pulse will have a higher intercavity intensity than a long pulse, and pulses of high enough intensity can undergo nonlinear interactions with the $\mathrm{Ti}: \mathrm{Al}_{2} \mathrm{O}_{3}$. This nonlinear interaction is exploited to produce high gain per roundtrip in the cavity.

For a further discussion of cavity modes, gain bandwidth, mode locking, and lasers in general, we recommend Lasers by A. Siegman ${ }^{23}$ and Lasers by P. Milonni and J. Eberly. ${ }^{24}$

We now discuss some of the properties of ultrashort pulses, particularly the relationship between the time domain and frequency domain descriptions of a pulse. In order to simplify our quantitative discussion, we first consider pulses with Gaussian envelopes. The Gaussian shape is convenient because the Fourier transform of a Gaussian function is another Gaussian function. It is important to note that the discussion we present can be easily generalized to any pulse shape, as shown below in the analysis section.

The electric field of a Gaussian pulse is given by

$$
E(t)=E_{0} \exp \left(-\frac{t^{2}}{2 \sigma_{t}^{2}}\right) \exp \left(-i \omega_{0} t\right),
$$

where $E_{0}$ is real, $\omega_{0}$ is the average, or "carrier,' frequency of the wave (near $10^{15} \mathrm{~Hz}$ for a $\mathrm{Ti}: \mathrm{Al}_{2} \mathrm{O}_{3}$ laser), and $\sigma_{t}$ is a measure of the pulse width in time. The full width at halfmaximum (FWHM) of the pulse duration is

$$
t_{\mathrm{FWHM}}=2 \sqrt{\ln (2)} \sigma_{t} .
$$

Note that this is the FWHM of the intensity of the pulse $\left(\propto E^{2}\right)$, not of the field.

We can write an equation completely analogous to Eq. (9) to describe the pulse in the frequency domain:

$$
\mathscr{E}(\omega)=\mathscr{E}_{0} \exp \left(-\frac{\left[\omega-\omega_{0}\right]^{2}}{2 \sigma_{\omega}^{2}}\right) .
$$

The FWHM of the intensity of the pulse's spectral width is given by

$$
\omega_{\mathrm{FWHM}}=2 \sqrt{\ln (2)} \sigma_{\omega} .
$$

From Eq. (2) we can calculate the "uncertainty" of our pulse in time and frequency domains. We identify the "uncertainty" as a measure of the pulse width in the particular domain. Using an equation analogous to Eq. (3) to calculate $\langle\omega\rangle$ and $\left\langle\omega^{2}\right\rangle$, we find

$$
\left\langle\omega^{2}\right\rangle=\omega_{0}^{2}+\frac{\sigma_{\omega}^{2}}{2}, \quad\langle\omega\rangle=\omega_{0},
$$

so that

$$
\Delta \omega=\frac{\sigma_{\omega}}{\sqrt{2}} .
$$

An identical expression holds for $\Delta t$. Thus Eq. (5) becomes

$$
\Delta \omega \Delta t=\frac{\sigma_{\omega} \sigma_{t}}{2} \geqslant \frac{1}{2}
$$

or

$$
\sigma_{\omega} \sigma_{t} \geqslant 1 \text {. }
$$

Thus 1 is the minimum time-bandwidth product of a Gaussian shaped pulse. It's important to realize that the minimum value given here depends on (i) the shape of the pulse envelope being Gaussian and (ii) the definition of $\Delta \omega$ and $\Delta t$ given above (as compared to, say, FWHM). A Gaussian pulse which meets the minimum time-bandwidth constraint is said to be "time-bandwidth limited," or "transform limited." In the case of ultrashort pulses, we hope to generate transform limited pulses, since we want the minimum pulse duration for a given spectral bandwidth.

This does, however, bring up the question: "Under what conditions won't a pulse be transform limited?' To explore the answer to this question, consider the oscillation of a spectral component, $\omega$, of the pulse: $\cos \left(k_{\omega} z-\omega t+\phi[\omega]\right)$, where $k_{\omega}$ is the wave number of the $\omega$ component. $\phi(\omega)$ is the component's phase which can be expanded as a Taylor series about the carrier frequency: 


$$
\begin{aligned}
\phi(\omega)= & \phi\left(\omega_{0}\right)+\left(\frac{d \phi}{d \omega}\right)_{\omega_{0}}\left(\omega-\omega_{0}\right) \\
& +\frac{1}{2 !}\left(\frac{d^{2} \phi}{d \omega^{2}}\right)_{\omega_{0}}\left(\omega-\omega_{0}\right)^{2}+\cdots
\end{aligned}
$$

(Please see the Appendix for the derivation and a physical discussion of this expression.) Pulses are transform limited if the coefficients of the quadratic and higher order terms are zero.

In general this won't be the case and the result is that, in the time domain, the short pulse will have a time dependent frequency. We are therefore motivated to more carefully consider Eq. (9). The harmonic term, $\exp \left(-i \omega_{0} t\right)$, is of limited validity as it stands because it describes only a single frequency pulse-a pulse which if described by Eq. (17) would have all terms of second order or higher equal to zero. Equation (9) can be made to describe a pulse with a time dependent frequency if we construct a more general expression for the argument of the exponent. We replace $\omega_{0} t$ with $g(t)$, where

$$
\begin{aligned}
g(t) & =g\left(t_{0}\right)+\left(\frac{d g}{d t}\right)_{t_{0}}\left(t-t_{0}\right)+\frac{1}{2 !}\left(\frac{d^{2} g}{d t^{2}}\right)_{t_{0}}\left(t-t_{0}\right)^{2}+\cdots \\
& =g_{0}+\omega_{0}\left(t-t_{0}\right)+\alpha\left(t-t_{0}\right)^{2}+\cdots
\end{aligned}
$$

It is possible to determine $g(t)$ for a given pulse, ${ }^{2,25,26}$ and from this the instantaneous frequency of the pulse as a function of time, since

$$
\omega(t)=\frac{d}{d t} g(t) .
$$

If all the terms beyond the linear terms in Eqs. (17) and (18) are zero, then the pulse is described by a single carrier frequency $\omega_{0}$ [consider Eq. (19)]. If any terms beyond the linear term are nonzero, then the pulse has a time dependent frequency and does not conform to the minimum constraint of the HUP.

In particular, if the quadratic term of the Taylor series is nonzero, the pulse is linearly dispersed, or "chirped," since the instantaneous frequency is linear in time [see Eq. (19)]. Accumulation of the quadratic term is physically due to the dispersion of the optical components (prisms, mirrors, lenses, beam splitters, etc.) with which the pulse interacts as it propagates. Control of this parameter, in both magnitude and sign, is a critical part of the technical operation of ultrashort laser pulse experiments. ${ }^{2,25,26}$ The terms beyond the quadratic become relatively more important as the quadratic term is minimized and are critical to reduce in order to drop the pulse duration below $100 \mathrm{fs}$.

In summary, a $\mathrm{Ti}: \mathrm{Al}_{2} \mathrm{O}_{3}$ laser is capable of generating a pulse with spectral bandwidth $\sigma_{\omega}$. Given this bandwidth, the minimum temporal duration of the pulse is given by the HUP. Thus, if one can generate transform limited pulses, laboratory experiments can be built which demonstrate and quantify this basic principle of quantum mechanics.

\section{EXPERIMENTAL SETUP}

We start this section with some words of caution. The peak of the $\mathrm{Ti}: \mathrm{Al}_{2} \mathrm{O}_{3}$ laser emission is in the near infrared, however, there is enough energy in the red that the beam can be seen by the unaided eye. Looking at the beam in this way, one might be led to believe that the laser is weak, as the visible portion of the emission is faint. This is incorrect. The laser has an average output power of $600 \mathrm{~mW}$ and a peak power of over $10^{5} \mathrm{~W}$. Significant and permanent retinal damage will occur if the beam enters the eye. Throughout all stages of the laboratory experiment, proper safety goggles should be worn by students and faculty.

The experimental layout, shown in Fig. 2, consists of an argon ion laser, $\mathrm{Ti}: \mathrm{Al}_{2} \mathrm{O}_{3}$ laser oscillator, external-prism pulse compressor, autocorrelator, and spectrometer. Central to the laboratory is the $\mathrm{Ti}: \mathrm{Al}_{2} \mathrm{O}_{3}$ laser ${ }^{27}$ pumped with an argon ion laser intensity stabilized at $4.5 \mathrm{~W}$ (all lines). The argon laser beam maintains a fixed propagation direction with an external pointing stabilizer. ${ }^{28}$ Maintaining spatial and pointing properties of the pump laser is critical in providing intensity and mode locking stability of the ultrashort pulse laser.

Alignment of the $\mathrm{Ti}: \mathrm{Al}_{2} \mathrm{O}_{3}$ oscillator is an important part of the laboratory, and we consider it to be the students' first experiment. The central concern in aligning the laser cavity is to minimize phase distortion, i.e., make all the nonlinear terms in Eqs. (17) and (18) zero. To aid in this, one can manipulate the "amount of glass" in the beam, i.e., the position of the compensation prisms along an axis that bisects the prism apex and is in a plane perpendicular to the prism surfaces. The prisms provide negative dispersion and can be used to control the magnitude and sign of the dispersion compensation within the laser cavity: More glass means more positive dispersion compensation. Mode locking stability is maintained when the overall cavity (compensation prisms plus gain medium and mirrors) has near zero net dispersion. This is achieved with the prisms set to balance the natural positive dispersion of the gain medium. ${ }^{2,26,29}$ Beyond the zero-dispersion condition, the prism material must be carefully chosen to minimize the third and fourth order phase distortion. Though the oscillator has a roundtrip dispersion balance, the output has a positive chirp caused, in part, by the pulse's passage through the output coupler. The zerochirp condition inside the cavity is designed to occur inside the gain medium in order to best effect Kerr lensing.

Once a stable mode locked output is attained from the oscillator, its spectrum and autocorrelation traces are measured as the second and third experiments carried out by the students. Measuring the spectrum is a good introduction into the physical properties of mode locked pulses and it helps to illustrate the relations given by the HUP. Mode locked pulses, having a short temporal duration, display a very broad spectrum compared to the continuous wave spectra of a single-mode laser. Broadband measurements are done using a linear-array-detector spectrometer. ${ }^{30}$ The spectrum can be read directly from the laser output (after heavy attenuation) or from a scrap reflection in the autocorrelator. Given the pulse spectrum, a quick, back-of-the-envelope calculation can give an idea of the pulse duration since $\Delta f$ $=\left(c / \lambda^{2}\right) \Delta \lambda$ and $\Delta t \approx 1 / \Delta f$.

Following the spectral measurements students embark on the more difficult pulse duration measurement. Output from the Ti: $\mathrm{Al}_{2} \mathrm{O}_{3}$ laser is filtered and sent through a double pass, external-prism-pair compressor to compensate for the chirp inherent in the laser output, from the output coupler and the IR pass filter, and from the beam splitters in the autocorrelator. Both external prisms are aligned at the Brewster angle and mounted on translation stages so they can maintain this 

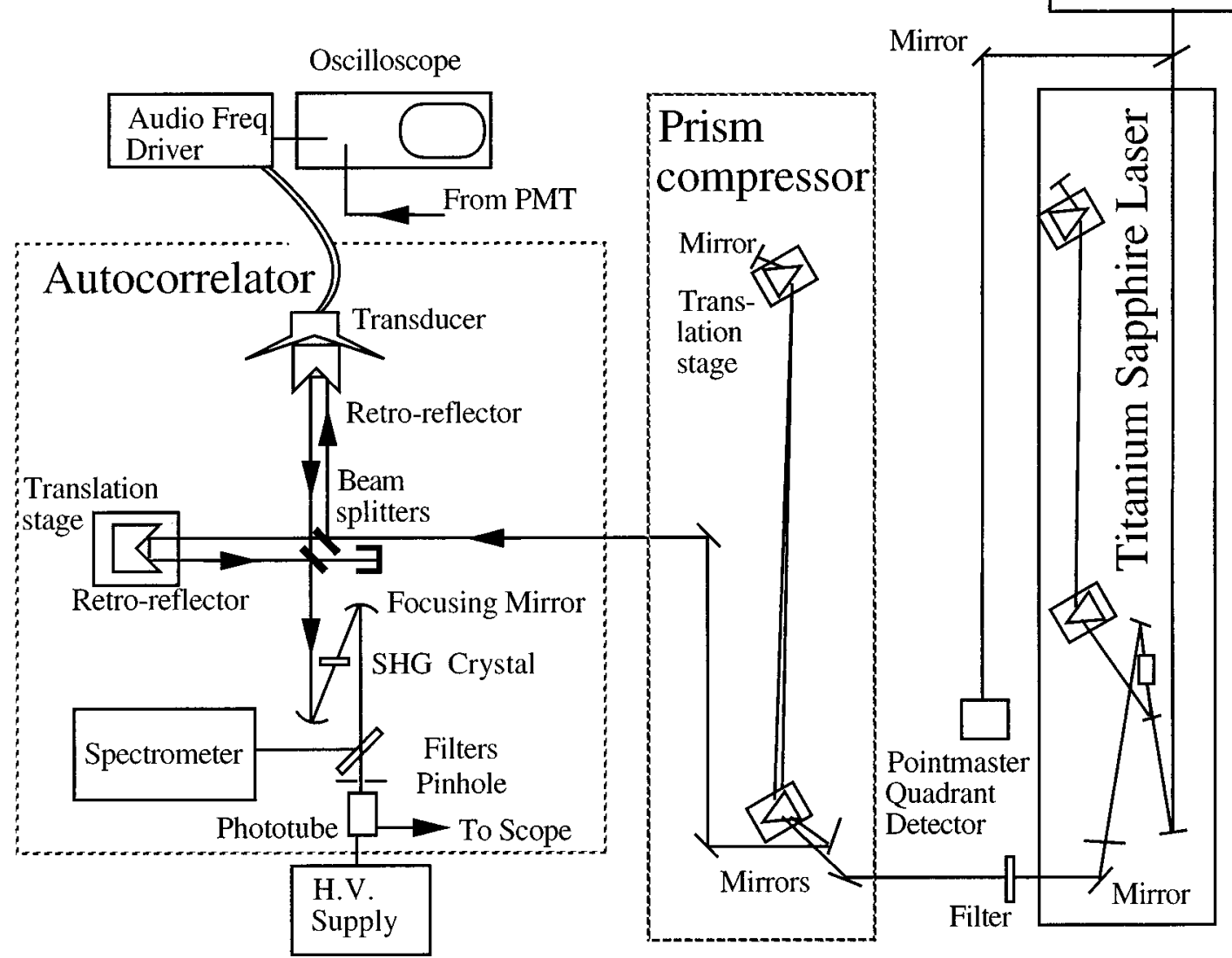

Fig. 2. Experimental setup.

alignment as they are translated through the beam. This configuration allows for adjustment of chirp, which leads to observable changes in the pulse correlation time, envelope ratio, and envelope shape. It is worth noting that we have found that the tip-to-tip prism separation is greater for the external prism pair than for the intercavity pair. This is indicative of the larger degree of "prechirp" needed to compensate for dispersion in the autocorrelator optics which the pulse will encounter. All mirrors used in this experiment are silver-coated substrate with a protective magnesium-fluoride layer. Silver is the preferable coating since it has a broad and flat reflective bandwidth over the wavelengths of interest, and thus does minimal modification of the spectral content of the pulse.

The autocorrelator design shown in Fig. 2 is based on previously reported configurations. ${ }^{26}$ The input beam is split into two beams with a front surface, $50 / 50$ beam splitter (back surface is antireflection coated). Each beam is retroreflected with aluminum mirror corner cubes and recombined with a second, separate beam splitter. The use of two beam splitters is important in that each path in the interferometer will contain the same amount of beam-splitter glass, and therefore the same amount of dispersion. Thus the externalprism pair is aligned to prechirp the pulse such that the minimum pulse width will be measured by the autocorrelator. Presumably, the minimum chirp occurs at the harmonic crystal where the correlation function is generated. The interference aspect of this technique depends critically on the align- ment of the beam splitters, and each is on a separate tilt mount. A quick check of the alignment can be made by looking at the beam overlap at a distant location (several meters) with an IR sensitive card. The combined beams are focused into a 50- $\mu \mathrm{m}$-thick second harmonic generation (SHG) KDP crystal $^{31}$ with a concave, silver-coated mirror (focal length $=10 \mathrm{~cm}$ ). The SHG is collected with a second concave mirror positioned to focus the light onto a photomultiplier tube (PMT). Several blue-pass filters are placed in front of the PMT to eliminate the fundamental, IR laser light. The photocurrent is converted into a voltage with a $1000-\Omega$ resistor and read on a digital oscilloscope.

One of the corner cubes is mounted on a fine translation stage adjusted for a match of the interferometer path lengths. The other corner cube is mounted on the cone of an audio speaker. By driving the speaker with the amplified triangle wave of a function generator the interference pattern is scanned at audio frequency repetition rates. This rate is tens of thousands times slower than the pulsing rate of the laser, so the PMT signal is approximately continuous throughout the scan. Too large a translation amplitude can reduce the interference fringe contrast, so care must be taken not to tune the audio frequency too close to a low frequency resonance of the speaker/cube system. Though audio speakers are designed for a relatively flat frequency response, the large aluminum housing of the corner cube glued to the cone will change these fine characteristics and low frequency resonances may develop. Precise position of the corner cube is 
not important in this technique since the physical translation is inferred from the fringe peaks. The separation between peaks corresponds to a net path change of one wavelength of the fundamental frequency, and this wavelength is known from the spectrum.

Relating the spectrum measured in the second experiment to the autocorrelation trace measured in the third is the subject of the following section.

\section{ANALYSIS}

This section describes the steps of analysis used to determine if a given laser pulse is at the limit of the HUP, i.e., whether or not it is transform limited. Starting with the power spectrum of the pulse, we take its square root to generate a spectral amplitude followed by a Fourier transform to generate the time amplitude (electric field) of the pulse. The square of the time amplitude is proportional to the intensity of the pulse, assuming that the pulse was originally transform limited. This assumption must be made because the square root of the spectrum, unfortunately, is not a complete description of the pulse in the frequency domain. It measures the intensity of each frequency component, but indicates nothing about each component's phase. To carry out a Fourier transformation from the frequency domain to the time domain we need to know the phase information, and this is not easily obtained. To work around this, we assume the phase of each frequency component is zero; in the context of Eq. (17), this is equivalent to setting $\phi(\omega)=0$, although, in principle, we can choose any constant value for $\phi\left(\omega_{0}\right)$. This assumption constrains the pulse to have the properties of a transform limited pulse. We numerically calculate the autocorrelation signal from the time domain description of the pulse and then compare this with the autocorrelation measurement of the actual pulse. If the two match, the pulse is transform limited, if not, there is a nonlinear phase associated with the frequency components of the pulse. Schematically, if $A$ represents amplitude and $I$ represents intensity (with the last step explained later):

\section{$I(\nu)$ (spectrum) \\ $\Downarrow$ square root}

$A(\nu)$

(assume each frequency component has zero phase)

$\Downarrow$ Fourier transform

$A(t)$

$\Downarrow_{\text {complex square }}$

$I(t)$

$\Downarrow$ numeric analysis

autocorrelation signal.

We have written an analysis program using Igor Pro, sold by Wavemetrics, Inc., which combines a powerful analysis package with high quality graphics. An Igor macro which follows this algorithm was used to generate the autocorrelation signal given the pulse spectrum as input and can be obtained directly from Tom Donnelly or by downloading from Carl Grossman's website, /http://laser.swarthmore.edu/ $\mathrm{html} /$ research/grossman/index.html.

Below we outline the theory relevant to the interferometric autocorrelation. ${ }^{32}$ As shown in Fig. 2, the pulse is split in the autocorrelator and the two resulting pulses pass collinearly through the KDP crystal. The pulses may be delayed with respect to one another so we represent their electric fields as $E\left(\omega_{0}, t\right)$ and $E\left(\omega_{0}, t-\tau\right)$, as defined in Eq. (9). These fields establish a nonlinear dipole moment, $P^{2 \omega_{0}}$, in the crystal at frequency $2 \omega_{0}$. The power series expansion of the dipole moment in terms of the field strength is ${ }^{33}$

$$
\begin{aligned}
P(t) & =\chi^{(1)} E(t)+\chi^{(2)} E^{2}(t)+\chi^{(3)} E^{3}(t)+\cdots \\
& =P^{0}(t)+P^{1 \omega}(t)+P^{2 \omega}(t)+\cdots,
\end{aligned}
$$

where the $\chi^{(i)}$ are the nonlinear optical susceptibility tensors. Therefore, the nonlinear dipole moment at $2 \omega_{0}$ is proportional to the square of the sum of the fields:

$$
P^{2 \omega_{0} \propto[E(t)+E(t-\tau)]^{2} .}
$$

The time averaged intensity of the KDP's emission at $2 \omega_{0}$, $\left\langle I^{2 \omega_{0}}\right\rangle$, is proportional to the complex square of the dipole at that frequency:

$$
\begin{aligned}
\left\langle I^{2 \omega_{0}}\right\rangle \propto & \left|P^{2 \omega_{0}}\right|^{2} \\
\propto & {\left[E(t)^{2}+E(t-\tau)^{2}+2 E(t) E(t-\tau)\right] } \\
& \times\left[E^{*}(t)^{2}+E^{*}(t-\tau)^{2}+2 E^{*}(t) E^{*}(t-\tau)\right] .
\end{aligned}
$$

Integrating this result from $t=-\infty$ to $t=+\infty$, for the Gaussian pulse of Eq. (9), we arrive at

$$
\begin{aligned}
\left\langle I^{2 \omega_{0}}(\tau)\right\rangle \propto & {\left[1+\exp \left(\frac{-\tau^{2}}{2 \sigma_{t}^{2}}\right)\left\{2+\cos \left(2 \omega_{0} \tau\right)\right\}\right.} \\
& \left.+4 \exp \left(\frac{-3 \tau^{2}}{8 \sigma_{t}^{2}}\right) \cos \left(\omega_{0} \tau\right)\right] .
\end{aligned}
$$

This is the interferometric autocorrelation signal measured at the photomultiplier tube in Fig. 2.

A similar result can be derived for Gaussian pulses which have a chirp $[\alpha \neq 0$ in Eq. (18)]. In this case,

$$
E(t)=E_{0} \exp \left(-\frac{t^{2}}{2 \sigma_{t}^{2}}\right) \exp \left[i\left(\omega_{0}+\alpha t\right) t\right] .
$$

By the same process we went through to arrive at Eq. (23), we find

$$
\begin{aligned}
\left\langle I^{2 \omega_{0}}(\tau)\right\rangle \propto & {\left[1+\exp \left(\frac{-\tau^{2}}{2 \sigma_{t}^{2}}\right)\left\{2+\cos \left(2 \omega_{0} \tau\right)\right.\right.} \\
& \left.\times \exp \left(-2(\sigma \alpha \tau)^{2}\right)\right\}+4 \exp \left(\frac{-3 \tau^{2}}{8 \sigma_{t}^{2}}\right) \\
& \left.\times \exp \left(-\frac{(\sigma \alpha \tau)^{2}}{2}\right) \cos \left(\omega_{0} \tau\right) \cos \left(\frac{\alpha \tau^{2}}{2}\right)\right] .
\end{aligned}
$$

We can work out the expression for $\left\langle I^{2 \omega_{0}}\right\rangle$ numerically for any pulse shape and with any order of phase distortion in time. This is done by defining an electric field

$$
E(t)=A(t) \exp \left(i\left[\omega_{0}+\alpha t+\beta t^{2}+\cdots\right] t\right)
$$



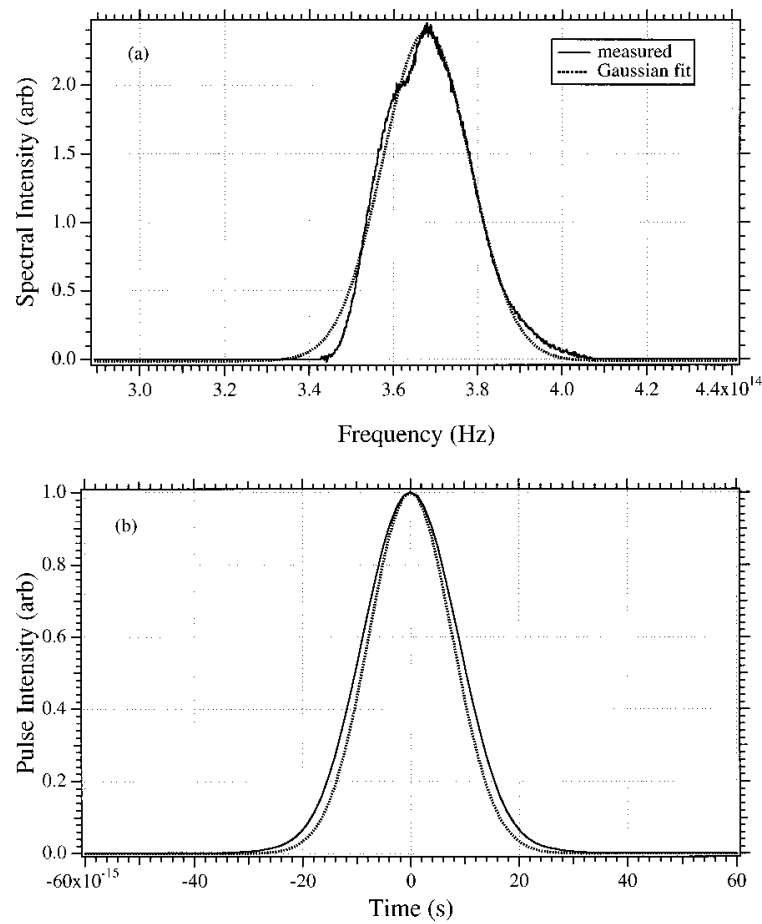

Fig. 3. (a) The spectrum of the laser and a Gaussian fit to the spectrum. The spectrum has a FWHM of $2.45 \times 10^{13} \mathrm{~Hz}$. (b) Through a Fourier transformation we can find the transform limited pulse intensity that is implied by the pulse spectrum (see the text). The solid curve in (b) is calculated from the measured spectrum and the dotted curve is calculated for the Gaussian fit to the spectrum. (-) FWHM=20 fs; ( $\cdots)$ FWHM $=18$ fs.

and a similar electric field delayed by a time $\tau, E(t-\tau)$, where $A(t)$ is the appropriate envelope function. The time averaged intensity of the KDP emission is then calculated numerically as described by Eqs. (21) and (22): We fix one field in time $(t=0)$ and then we calculate the square of the nonlinear dipole moment at every $\tau(-\infty \leqslant \tau \leqslant+\infty)$, giving $\left\langle I^{2 \omega_{0}}(\tau)\right\rangle$. The algorithm for this numeric calculation can be used to calculate the appropriate autocorrelation signal for any spectrum that is initially measured. There is no longer a need to assume that the pulse is Gaussian or otherwise. This analysis consists of three basic procedures that can be executed in most computer data analysis programs. (1) A data transformation procedure prepares the raw data, an array of spectral intensities and corresponding frequencies, into a field amplitude with appropriate array dimensions and frequency interval for a fast Fourier transform (FFT). (2) FFT converts the field amplitude spectrum to the complex, transform limited pulse envelope of the field amplitude. The magnitude of this complex envelope is used in the third procedure. (3) A simulation of the autocorrelation data is made by calculating the square of Eq. (21), the nonlinear optical polarization, for a set of interferometric time delays.

\section{RESULTS AND DISCUSSION}

Typical data for the experiments described above are shown in Figs. 3 and 4. The spectrum of a short pulse is shown in Fig. 3(a) and the implied transform limited pulse intensity is shown in Fig. 3(b). We have fit the spectrum to a Gaussian envelope and calculated the corresponding transform limited pulse intensity for comparison since this will represent the pulse of minimum possible duration. The auto-

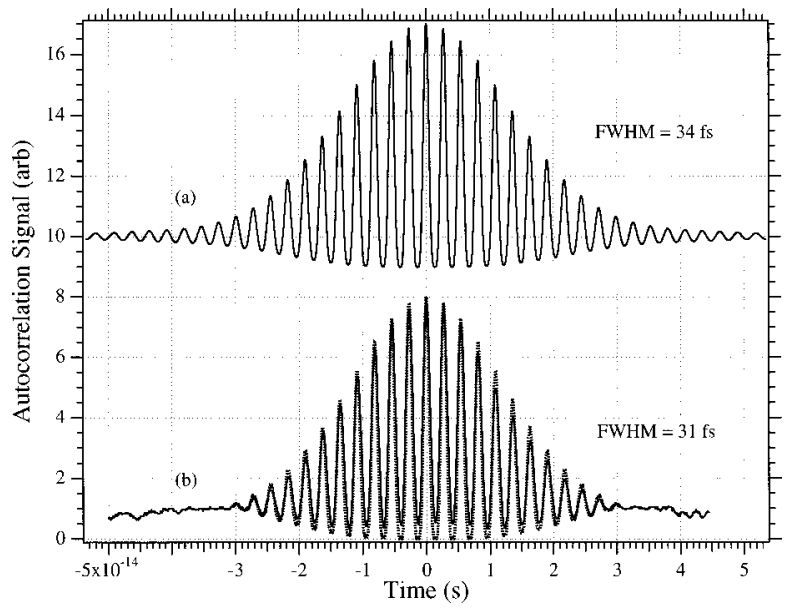

Fig. 4. Autocorrelation signals: (a) numeric result when experimental spectrum is input; (b) the solid line is the measured signal and the dotted line is the numeric result when a Gaussian approximation of the experimental spectrum [see Fig. 3(a)] is used as input. The FWHM of the pulse is a factor of 1.7 smaller than the FWHM of autocorrelation (Gaussian pulses), therefore the FWHM of the pulse intensity is (a) $20 \mathrm{fs}$, (b) $18 \mathrm{fs}$.

correlation traces that are generated from the pulses in Fig. 3 (b) are shown in Fig. 4. Here we compare the measured autocorrelation trace [corresponding to the measured spectrum of Fig. 3(a)] with that which is calculated numerically from both the measured spectrum and the Gaussian fit to the spectrum. We assign a time axis to the measured autocorrelation trace by noting that the interference fringes are separated by one period of oscillation. The period of oscillation can be determined from the peak of the spectrum, e.g., Fig. 3 (a) shows the spectral peak at $3.68 \times 10^{14} \mathrm{~Hz}$, corresponding to a period of $2.72 \mathrm{fs}$.

It is evident that the autocorrelation trace numerically generated from the measured spectrum is slightly broader than that which is actually measured. This results from the limitation of our spectrum analyzer. The response of the detector in the spectrum analyzer is not flat as a function of frequency and thus distorts the actual spectrum. Further, the detector resolution is such that we are unable to generate a signal to noise ratio of greater than 100; this limits our ability to gain information about the spectral wings. To overcome this, we assume that the spectral intensity is zero beyond what we can reliably measure in the spectral wings. Further, since the time resolution of the fast Fourier transform in the numeric analysis is equal to the inverse of the total spectral frequency, a time resolution on the order of $1 \mathrm{fs}$ requires a bandwidth $>10^{14} \mathrm{~Hz}$. To achieve this bandwidth, we truncate the spectrum as described above and then add zeros to the measured spectrum below approximately $3.4 \times 10^{14} \mathrm{~Hz}$ and above approximately $4.1 \times 10^{14} \mathrm{~Hz}$. These problems with the spectrum will be avoided in the future by taking high signal to noise measurements well into the spectral wings using a spectrometer and calibrated PMT. The distortion of the spectrum we input into the numeric analysis program accounts for the generated autocorrelation trace being slightly broader than the measured one.

The curves of Figs. 3(a) and 4(b) show that if a Gaussian shape is assumed for the spectrum the resulting numeric autocorrelation trace is nearly identical to the measured trace [see Fig. 4(b)]. This is evidence that, although our spectral 


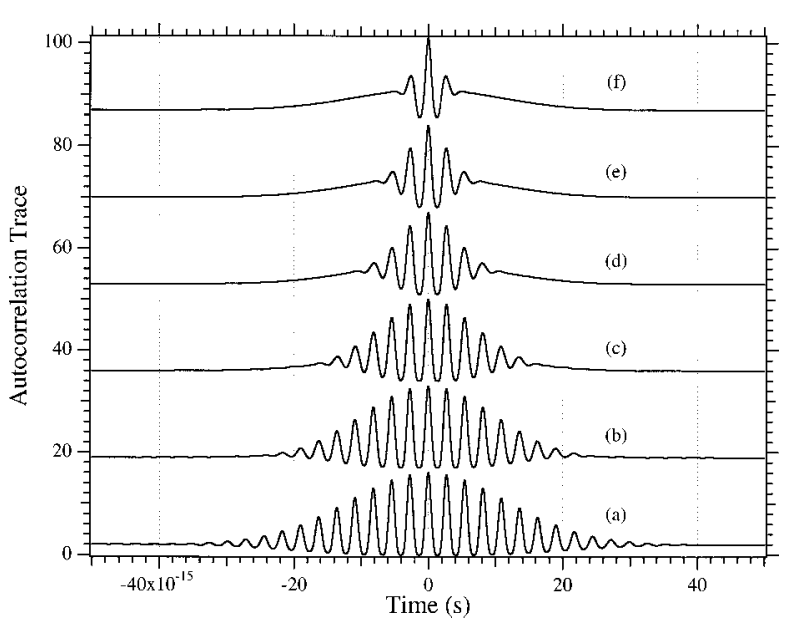

Fig. 5. Autocorrelation traces generated numerically for a Gaussian pulse $\left(\sigma_{t}=10.8 \mathrm{fs}\right)$ with the following values of chirp [see Eqs. (24) and (25)]: (a) $\alpha=0.0 \mathrm{~s}^{-2}$, (b) $\alpha=0.005 \mathrm{~s}^{-2}$, (c) $\alpha=0.01 \mathrm{~s}^{-2}$, (d) $\alpha=0.02 \mathrm{~s}^{-2}$, (e) $\alpha$ $=0.03 \mathrm{~s}^{-2}$, (f) $\alpha=0.05 \mathrm{~s}^{-2}$.

measurements are imperfect, the pulse is transform limited. From Eq. (23) one can show that the FWHM of the autocorrelation trace, $t_{\mathrm{FWHM}-\mathrm{AC}}$ is related to $\sigma_{t}$ by

$$
t_{\mathrm{FWHM}-\mathrm{AC}}=(2 \sqrt{ } 2) \sigma_{t} .
$$

Thus from the measured autocorrelation trace in Fig. 4(b) we find $\sigma_{t}$ to be 10.8 fs. This is exactly $\sigma_{t}$ derived in Fig. 3(b) for the Gaussian pulse intensity, which was, in turn, calculated from the spectrum shown in Fig. 3(a). From Eq. (16) we calculate $\sigma_{\omega}=9.26 \times 10^{13} \mathrm{~Hz}$ or $\sigma_{f}=1.47 \times 10^{13} \mathrm{~Hz}$, and this is what we measure for the spectrum in Fig. 3(a). Thus the frequency and time descriptions of the measured pulse are at the minimum constraint of the HUP.

It should be pointed out that the autocorrelation trace of a transform limited pulse is easily identified. Figure 5 compares the autocorrelation traces [see Eq. (25)] that would be generated by a Gaussian pulse with varying degrees of chirp. The transform limited $(\alpha=0)$ autocorrelation trace is shown in Fig. 5(a) and is identical to the trace of Fig. 4(b). We then add increasing amounts of chirp up through Fig. 5(f); in all cases third order and higher phase perturbations are zero. The distorting effects of chirp on the autocorrelation trace are evident: As the time delay between the correlating pulses is swept, different frequencies in each pulse interfere and thus the single frequency interference pattern of trace (a) is wiped out. Autocorrelation traces corresponding to nontransform limited pulses can be identified because they lack oscillatory behavior in the trace's wings. A casual observer may identify trace (f) as corresponding to a shorter duration pulse than trace (a) since, at a glance, it appears to have smaller FWHM. In fact, trace (a) corresponds to the shorter, transform limited pulse.

\section{CONCLUSION}

We have presented experiments which introduce students to some of the techniques and technology used in ultrafast science. The experiments demonstrate a connection between ultrashort laser pulses and quantum mechanics, and introduce students to numeric analysis and optics. We have worked out the mathematical details for the analysis of the experiments, and have included a numeric model which may be of use. We expect these experiments to be useful in training students for future endeavors, both academic and technology related.

\section{ACKNOWLEDGMENTS}

This work was generously supported by the National Science Foundation's Division of Undergraduate Education through Grant No. DUE 9650431 and the DuPont Aid to Education Program. We gratefully acknowledge the assistance of Professor Margaret Murnane in the operation and characterization of the Ti: $\mathrm{Al}_{2} \mathrm{O}_{3}$ laser and Professor John Townsend for his valuable discussions.

\section{APPENDIX}

Consider a pulse at the plane $z=0$. The field of the pulse can be approximated as ${ }^{25}$

$$
E(0, t)=A(t) \exp [i \psi(t)] \exp \left[-i \omega_{0} t\right],
$$

where $A$ is the real amplitude and $\psi$ is the phase modulation term. The approximation is valid as long as the range of the frequency component which make up the pulse is small compared to $\omega_{0}$. We let the pulse propagate through an arbitrary system and calculate the description of the output pulse at some time $t^{\prime}$. This is done by taking the Fourier transform of Eq. (A1), multiplying by $\exp [i \phi(\omega)]$, where $\phi(\omega)$ is the phase shift through the system, and then taking the inverse Fourier transform:

$$
\begin{aligned}
E_{\text {out }}\left(t^{\prime}\right)= & \int \frac{d \omega}{2 \pi} \exp \left[-i \omega t^{\prime}\right] \exp [i \phi(\omega)] \\
& \times \int d t \exp [i \omega t] A(t) \exp [i \psi(t)] \exp \left[-i \omega_{0} t\right]
\end{aligned}
$$

Knowing the form of the input pulse, we can calculate the description of the output pulse if we know $\phi(\omega)$.

Physically, $\phi(\omega)$ accounts for the phase accumulated, relative to the carrier frequency, by each frequency component as the pulse propagates through the system:

$$
\phi(\omega)=\left(k_{\omega}-k_{\omega_{0}}\right) z .
$$

Thus $\phi(\omega)=0$ if the pulse propagates through vacuum. Given that the propagation constants are expressed as $k_{\omega}$ $=n_{\omega} \omega / c$ and that the refractive index, $n_{\omega}$, can be expanded in a Taylor series about $\omega_{0}$, the phase term is expressed as

$$
\begin{aligned}
\phi(\omega)= & \frac{z}{c}\left[\omega n_{\omega}-\omega_{0} n_{\omega_{0}}\right] \\
= & \frac{z}{c}\left[\omega \left\{n_{\omega_{0}}+\left.\frac{d n_{\omega}}{d \omega}\right|_{\omega_{0}}\left(\omega-\omega_{0}\right)+\left.\frac{1}{2 !} \frac{d^{2} n_{\omega}}{d \omega^{2}}\right|_{\omega_{0}}\right.\right. \\
& \left.\left.\times\left(\omega-\omega_{0}\right)^{2}+\cdots\right\}-\omega_{0} n_{\omega_{0}}\right] .
\end{aligned}
$$

If the index of refraction is accurately known as a function of frequency for the materials through which the beam will propagate, then Eq. (A4) allows the calculation of the coefficients of the expansion of $\phi(\omega)$ shown in Eq. (17). The physical significance of each expansion coefficient in Eq. 
(17) is now more apparent. For example, from Eq. (A4), it can be shown that

$$
\left(\frac{d \phi(\omega)}{d \omega}\right)_{\omega_{0}}=\frac{z}{c}\left(n_{\omega_{0}}+\omega_{0}\left(\frac{d n_{\omega}}{d \omega}\right)_{\omega_{0}}\right)
$$

and thus that this coefficient in Eq. (17) represents the group delay of the entire output pulse. The term of next highest order, which is associated with chirp in the output pulse, can be similarly determined. Chirp results because each component of the pulse travels through a dispersive medium at a different speed, thus increasing the temporal duration of the pulse and resulting in a time dependent frequency. It is interesting to note that, like the first order term, the second order term results in an overall group delay in addition to the chirp.

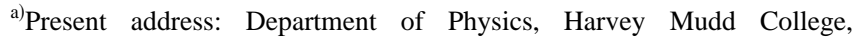
Claremont, California 91711; electronic mail: Tom_Donnelly@hmc.edu

${ }^{1}$ S. L. Shapiro, "Introduction-A Historical Overview," in Ultrashort Light Pulses, Picosecond Techniques and Applications, edited by S. L. Shapiro (Springer-Verlag, Berlin, 1977), pp. 1-15.

${ }^{2}$ R. L. Fork, C. H. Brito Cruz, P. C. Becker, and C. V. Shank, "Compression of optical pulses to six femtoseconds by using cubic phase compensation," Opt. Lett. 12, 483-485 (1987).

${ }^{3}$ J. Zhou, G. Taft, C. P. Huang, M. M. Murnane, and H. C. Kapteyn, "Pulse evolution in a broad-bandwidth Ti: sapphire laser,' Opt. Lett. 19, 11491151 (1994); A. Baltuska, Z. Wei, M. Pschenichnikov, and D. Wiersma, "Optical pulse compression to 5 fs at a $1-\mathrm{MHz}$ repetition rate,", ibid. 22, 102-104 (1997).

${ }^{4} \mathrm{An}$ excellent resource for researching current and past work in this field is the Ultrafast Phenomena conference proceedings published in the Springer Series in Chemical Physics by Springer-Verlag.

${ }^{5}$ R. W. Schoenlein, L. A. Peteanu, R. A. Mathies, and C. V. Shank, "The First Step in Vision: Femtosecond Isomerization of Rhodopsin," Science 254, 412-415 (1991).

${ }^{6}$ N. K. Boardman, "The Photochemical Systems of Photosynthesis," in Advances in Enzymology, edited by F. F. Nord (Interscience, New York, 1968), pp. 1-79.

${ }^{7}$ T. Foerster, in Modern Quantum Chemistry, edited by O. Sinanoglu (Academic, New York, 1965), Part III, pp. 93-137.

${ }^{8}$ S. L. Shapiro, A. J. Campillo, V. H. Kollman, and W. B. Goad, "Exciton Transfer in DNA," Opt. Commun. 15, 308-310 (1975).

${ }^{9}$ C. W. Rella, A. Kwok, K. Rector, J. Hill, H. Schwettman, D. Dlott, and M. Fayer, "Vibrational Echo Studies of Protein Dynamics," Phys. Rev. Lett. 77, 1648-1651 (1996).

${ }^{10}$ A. L'Huillier, L. Lompre, G. Mainfray, and C. Manus, “High Order Harmonic Generation in Rare Gases,' in Atoms in Intense Laser Fields, edited by M. Gavrilla (Academic, Boston, 1992), pp. 139-206.

${ }^{11}$ J. J. Macklin, J. D. Kmetec, and C. L. Gordon III, "High-Order Harmonic Generation Using Intense Femtosecond Pulses,' Phys. Rev. Lett. 70, 766769 (1993)

${ }^{12}$ M. Murnane, H. Kapteyn, M. Rosen, and R. Falcone, "Ultrafast X-ray Pulses from Laser-Produced Plasmas,'” Science 251, 531-536 (1991).

${ }^{13} \mathrm{~K}$. Weninger, B. Barber, and S. J. Putterman, "Pulsed Mie Scattering Measurements of the Collapse of a Sonoluminescing Bubble," Phys. Rev. Lett. 78, 1799-1802 (1997).

${ }^{14}$ M. Nisoli, S. Stagira, S. De Silvestri, A. Stella, P. Tognini, P. Cheyssac, and R. Kofman, "Ultrafast Electronic Dynamics in Solid and Liquid Gallium Nanoparticles," Phys. Rev. Lett. 78, 3575-3578 (1997); W. H.
Knox, D. S. Chemla, G. Livescu, J. E. Cunningham, and J. E. Henry, "Femtosecond Carrier Thermalization in Dense Fermi Seas," ibid. 61, 1290-1293 (1988)

${ }^{15}$ L. Dhar, J. A. Rogers, and K. A. Nelson, "Time-Resolved Vibrational Spectroscopy in the Impulsive Limit," Chem. Rev. 94, 157 (1994); H. L. Fragnito, J.-Y. Bigot, P. C. Becker, and C. V. Shank, "Evolution of the Vibronic Absorption Spectrum in a Molecule Following Impulsive Excitation with a 6 fs Optical Pulse," Chem. Phys. Lett. 160, 101-104 (1989).

${ }^{16} \mathrm{G}$. M. Carter, "Excited-state dynamics and temporally resolved nonresonant nonlinear-optical processes in polydiacetylenes,'” J. Opt. Soc. Am. B 4, 1018-1024 (1987); T. Kobayashi, M. Yoshizawa, U. Stamm, M. Taiji, and M. Hasegawa, "Relaxation dynamics of photoexcitations in polydiacetylenes and polythiophene," ibid. 7, 1558-1578 (1990) (Special Edition on Ultrafast Spectroscopy of Chemical and Biological Processes).

${ }^{17} \mathrm{~A}$. M. Weiner and J. P. Heritage, "Picosecond and femtosecond Fourier pulse shape synthesis,' Rev. Phys. Appl. 22, 1619-1628 (1987).

${ }^{18}$ D. J. Griffiths, Introduction to Quantum Mechanics (Prentice-Hall, Englewood Cliffs, NJ, 1995), p. 108.

${ }^{19}$ In Ref. 18, p. 113

${ }^{20} \mathrm{~J}$. S. Townsend, A Modern Approach to Quantum Mechanics (McGrawHill, New York, 1992), pp. 115-119.

${ }^{21}$ P. F. Moulton, "Tunable Solid-State Lasers," Proc. IEEE 80, 348-364 (1992); P. M. W. French, "Ultrafast solid-state lasers," Contemp. Phys. 37, 283-301 (1996).

${ }^{22}$ F. Krausz, M. Ferman, T. Brabec, P. Curley, M. Hofer, M. Ober, C. Spielmann, E. Wintner, and A. Schmidt, "Femtosecond Solid State Lasers," IEEE J. Quantum Electron. 28, 2097-2122 (1992); T. Brabec, P. Curley, C. Spielmann, E. Wintner, and A. Schmidt, "Hard-aperture Kerr-lens mode locking," J. Opt. Soc. Am. B 10, 1029-1034 (1993).

${ }^{23}$ A. Siegman, Lasers (University Science Books, Mill Valley, 1986).

${ }^{24}$ P. Milonni and J. Eberly, Lasers (Wiley, New York, 1988).

${ }^{25}$ E. B. Treacy, "Optical Pulse Compression With Diffraction Gratings," IEEE J. Quantum Electron. 5, 454-458 (1969).

${ }^{26}$ C. P. J. Barty, B. E. Lemoff, and C. L. Gordon III, “'Generation, measurement, and amplification of 20-fs high-peak-power pulses from a regeneratively initiated self-mode locked Ti: sapphire laser," in Ultrafast Pulse Generation and Spectroscopy [Proc. SPIE 1861, 6-30 (1993)]. Also see, W. H. Knox, N. M. Pearson, K. D. Li, and C. A. Hirlimann, "Interferometric measurements of femtosecond group delay in optical components," Opt. Lett. 13, 574-576 (1988).

${ }^{27}$ Kapteyn-Murnane Laboratories L. L. C., model TS laser kit. This is an easy to construct, well-documented and excellently priced kit. Its current cost is $\$ 15,799$.

${ }^{28}$ Clark MXR, Inc.'s Pointmaster 4-axis Pointing and Positioning Stabilizer, model BPS-2. This unit operates with two four-quadrant photodetectors which feedback the argon laser's position to two piezos which, in turn, adjust steering mirrors. All control electronics are included. Its current price is $\$ 9800$.

${ }^{29}$ R. L. Fork, O. E. Martinez, and J. P. Gordon, “Negative dispersion using pairs of prisms," Opt. Lett. 9, 150-152 (1984).

${ }^{30} \mathrm{We}$ use Imaging and Sensing Technology's Laser Spectrum Analyzer, model E201. It is sensitive from 350 to $1100 \mathrm{~nm}$, scans at $18 \mathrm{~Hz}$, and currently costs $\$ 4396$.

${ }^{31}$ We bought our KDP crystal from Cleveland Crystals, Inc.

${ }^{32}$ K. L. Sala, G. A. Kenney-Wallace, and G. E. Hall, "CW Autocorrelation Measurements of Picosecond Laser Pulses," IEEE J. Quantum Electron. 16, 990-996 (1980); J. M. Diels, J. J. Fontaine, I. C. McMichael, and F. Simoni, "Control and measurement of ultrashort pulse shapes (in amplitude and phase) with femtosecond accuracy,"' Appl. Opt. 24, 1270-1282 (1985).

${ }^{33}$ R. W. Boyd, Nonlinear Optics (Academic, Boston, 1992).

\section{THE PERMANENCE OF MATHEMATICAL ACHIEVEMENT}

What we do may be small, but it has a certain character of permanence; and to have produced anything of the slightest permanent interest, whether it be a copy of verses or a geometrical theorem, is to have done something utterly beyond the powers of the vast majority of men.

G. H. Hardy, A Mathematician's Apology (Cambridge University Press, 1969; reprint of 1940 edition), p. 76. 